\title{
Vigilância Ambiental: Análise do Fornecimento de Água para Consumo Humano
}

\author{
Environmental Surveillance: Analysis of Water Supply for Human Consumption
}

Vigilancia Ambiental: Análisis del Suministro de Agua Para el Consumo Humano

Claudio José dos Santos Júnior ${ }^{1}$

Jackson Pinto Silva ${ }^{2}$

John Victor dos Santos Silva ${ }^{3}$

Alenilza Bezerra Costa ${ }^{4}$

Vânia Nascimento Tenório Silva ${ }^{5}$

Tâmara Moraes Bastos ${ }^{6}$

\section{Resumo}

Objetivo: Analisar a situação da vigilância dos parâmetros de qualidade e potabilidade da água para consumo humano no estado de Alagoas, Brasil. Método: Trata-se de um estudo exploratório, cunho quantitativo. As informações referentes à caracterização das formas de abastecimento de água e das condições do monitoramento da qualidade da água para consumo humano em Alagoas foram obtidas a partir dos relatórios do Programa Nacional de Vigilância da Qualidade da Água. Os dados foram analisados por estatística descritiva e apresentados através gráficos, tabelas e mapas. A discussão ocorreu à luz da literatura e legislação especializada. Resultados: Observou-se que a maioria das residências em Alagoas é abastecida por fornecimento coletivo de água do tipo SAA, o quantitativo de abastecimentos por SAA sem tratamento no estado é superior ao da região Nordeste e três

${ }^{1}$ Graduando do curso de Médica da Universidade Estadual de Ciências da Saúde de Alagoas (UNCISAL). Autor correspondente: Rua Dr. Jorge de Lima. No 113. Trapiche da Barra. 57072-900. Maceió, Al, Brasil. E-mail: claudiosantos_al@hotmail.com

${ }^{2}$ Mestre em Análise de Sistemas Ambientais pelo Centro Universitário CESMAC. Docente do Ensino Básico e Técnico do IFAL Maceió.

${ }^{3}$ Graduando do curso de Enfermagem da UNCISAL.

${ }^{4}$ Enfermeira. Docente da UNCISAL.

${ }^{5}$ Mestrado em Química e Biotecnologia pela UFAL. Docente do Ensino Básico e Técnico do (IFAL).

${ }^{6}$ Doutora em Ciências da Educação pela Universidade Tecnológica Intercontinental. Mestre em Educação Brasileira pela Universidade Federal de Alagoas. Especialista em Tecnologia Química. Professora de Química do IFAL.

Recebido: Ago./2018 - Aceito: Dez./2018. 
vezes maior que o cenário nacional. Foram verificados altos índices de Hepatite A, que chegam a superar a taxa de incidência brasileira para essa doença em 50\%. O percentual de análises mínimas para os parâmetros Cloro Residual Livre, Coliformes Totais e Turbidez ficou abaixo do estabelecido pela legislação para período em estudo. Conclusão: Ficou evidente a necessidade de fortalecimento das ações do setor de saúde e dos responsáveis pelos sistemas de fornecimento no sentido de implantar as diretrizes em vigor e aperfeiçoar o monitoramento dos parâmetros de controle, a fim de tornar o recurso fornecido à população de Alagoas mais seguro e com qualidade suficiente para prevenção de agravos diversos.

\section{Descritores: Água; Vigilância}

Sanitária Ambiental; Conservação dos Recursos Naturais.

\section{Abstract}

Objective: The objective of this work was to analyze the situation of the surveillance of water quality and potability parameters for human consumption in the state of Alagoas, Brazil. Method: This is an exploratory, documentary and quantitative study.
The information regarding the characterization of the water supply forms and the conditions of the monitoring of the quality of the water for human consumption in Alagoas were obtained from the reports of the National Program of Surveillance of Water Quality. Data were analyzed by descriptive statistics and presented through graphs, tables and maps. The discussion took place in the light of literature and specialized legislation. Results: It was observed that the absolute majority of the residences in Alagoas is supplied by collective supply of SAA type water. It was evidenced that the amount of untreated SAA supplies in the state is higher than in the Northeast region and three times higher than the national scenario. High rates of Hepatitis A were verified, which surpassed the Brazilian incidence rate for this disease in 50\%. The percentage of minimum analyzes for the parameters Free Residual Chlorine, Total Coliforms and Turbidity was below that established by the legislation for the period under study. Conclusion: It was evident the need to strengthen the actions of the health sector and those responsible for the supply systems in order to implement the current guidelines and improve the monitoring of control parameters, in order to make 
the resource provided to the population of Alagoas more safe and of sufficient quality to prevent various diseases.

\section{Descriptors: Water; Environmental} Health Surveillance; Conservation of Natural Resources.

\section{Resumen}

Objetivo: Este trabajo tuvo como objetivo analizar la situación de la vigilancia de los parámetros de calidad y potabilidad del agua para consumo humano en el estado de Alagoas, Brasil. Método: Se trata de un estudio exploratorio, del tipo documental y de cuño cuantitativo. Las informaciones referentes a la caracterización de las formas de abastecimiento de agua y de las condiciones del monitoreo de la calidad del agua para consumo humano en Alagoas se obtuvieron a partir de los informes del Programa Nacional de Vigilancia de la Calidad del Agua. Los datos fueron analizados por estadística descriptiva y presentados a través de gráficos, tablas y mapas. La discusión ocurrió a la luz de la literatura y legislación especializada. Resultados: Se observó que la mayoría absoluta de las residencias en Alagoas es abastecida por suministro colectivo de agua del tipo SAA. Se evidenció que el cuantitativo de abastecimientos por SAA sin tratamiento en el estado es superior al de la región Nordeste y tres veces mayor que el escenario nacional. Se verificaron altos índices de Hepatitis $A$, que llegan a superar la tasa de incidencia brasileña para esa enfermedad en un 50\%. El porcentaje de análisis mínimos para los parámetros Cloro Residual Libre, Coliformes Totales y Turbidez quedó por debajo de lo establecido por la legislación para el período en estudio. Conclusión: quedó evidente la necesidad de fortalecer las acciones del sector de salud y de los responsables de los sistemas de suministro para implantar las directrices en vigor $y$ perfeccionar el monitoreo de los parámetros de control, a fin de hacer el recurso proporcionado a la población de Alagoas más seguro y con calidad suficiente para la prevención de agravios diversos.

\section{Descriptores: Vigilancia Sanitaria Ambiental; Concervación de los Recursos Naturales.}

\section{Introdução}

A importância da água para a existência de vida na Terra é indiscutível $^{(1)}$. Trata-se de um recurso 
natural constitucional para a vida e de fundamental relevância para o desenvolvimento de diversas atividades antrópicas, tais como a produção de alimentos, de energia, de bens de consumo, de transporte e de lazer, assim como para a manutenção e o equilíbrio ambiental dos ecossistemas terrestres ${ }^{(2)}$.

Os efeitos na qualidade e na quantidade da água disponível, relacionados com o rápido crescimento da população mundial e com a concentração dessa população em grandes centros urbanos podem afetar, e até mesmo inviabilizar, a vida humana e o desenvolvimento econômico e social de uma região ${ }^{(3)}$.

Em situações onde predomina a falta de um sistema de abastecimento de água adequado, a água pode trazer riscos à saúde, o que implica negativamente na sua qualidade e uma maior probabilidade de transmissão de vetores e infecções ${ }^{(4)}$. Essas contaminações representam um dos principais riscos à saúde pública, sendo amplamente conhecida a estreita relação entre a qualidade de água e inúmeros agravos que acometem as comunidades, especialmente aquelas que não são atendidas por um serviço de saneamento básico de qualidade em seus locais de $\operatorname{moradia}^{(1)}$.

Nesse sentido, a preocupação descrita com relação à água motivou o desenvolvimento deste artigo, que tem por objetivo analisar a situação da vigilância dos parâmetros de qualidade e potabilidade da água para consumo humano no estado de Alagoas, expondo informações acerca das formas de abastecimento de água e das condições do monitoramento da qualidade deste recurso no Estado.

\section{Método}

\section{Caracterização da Área em Estudo}

O estado de Alagoas (AL) possui uma área de 27.779,343 $\mathrm{km}^{2}$, divididos em 102 municípios e uma população recenseada estimada em 3.120 .494 habitantes $^{(5)}$. O abastecimento de água em 76 municípios do estado é de responsabilidade da Companhia de Abastecimento de Água e Saneamento do Estado de Alagoas ${ }^{(6)}$.

Os municípios que não são atendidos pela companhia do estado, dispõem de abastecimento de água próprio e baseados em formas alternativas de fornecimento, tais como poços, cisternas e cacimbas. A água tratada pela companhia do estado é proveniente do Rio São Francisco, sendo este o único manancial com vazão segura em toda a região do 
semiárido alagoano. A água proveniente desse manancial é tratada e distribuída para os sistemas coletivos de abastecimentos que englobam duas ou mais cidades, interligadas por uma série de adutoras. Em algumas situações o tratamento ocorre junto à área urbana, aduzindo-se água bruta. Em outras se tem Estações de Tratamento de Água (ETA), aduzindo água já tratada ${ }^{(6)}$.

\section{Coleta dos Dados}

Trata-se de um estudo exploratório, do tipo documental e de cunho quantitativo. A pesquisa documental foi realizada utilizando os dados dos relatórios de avaliação da vigilância da qualidade da água no estado de Alagoas do Programa Nacional de Vigilância da Qualidade da Água para Consumo Humano VIGIAGUA. A fonte primária das informações do VIGIAGUA é o Sistema de Informação de Vigilância da Qualidade da Água para Consumo Humano - SISAGUA, relativas ao ano de 2011 e publicado no ano de $2012^{(7)}$. Foram extraídas, ainda, informações sobre o número de diagnósticos confirmados de Hepatite A no estado através do Sistema de Informação de Agravos de Notificação ${ }^{(8)}$.
O SISAGUA é um sistema de informação em saúde tem por objetivo manter atualizado um banco de dados com informações referentes às diferentes formas de Abastecimento de Água no âmbito nacional. Os relatórios consultados constituem uma das ações da Secretaria Nacional de Vigilância em Saúde, que é um órgão do Ministério da Saúde, e se referem as informações mais atualizadas disponibilizados pelo órgão sobre o referido tema para $o$ estado de Alagoas ${ }^{(9)}$.

Os dados foram agrupados em banco de dados por meio do programa Microsoft Excel 2017 e analisados por meio de estatística descritiva de frequência absoluta e relativa, e apresentados através de gráficos, tabelas e mapas. A discussão dos dados foi realizada a luz da literatura especializada sobre o tema, que incluiu a consulta a normativos que estabelecem os padrões sobre 0 abastecimento e distribuição da água para consumo em âmbito nacional e alguns trabalhos científicos que abordam temas relacionados ao assunto em estudo.

\section{Aspectos Éticos}

Por se tratar de pesquisa envolvendo apenas dados secundários, 
extraídos de relatórios técnicos de domínio público, não houve a necessidade de submissão ao Comitê de Ética em Pesquisa, nem de firmar Termo de Consentimento Livre e Esclarecido.

\section{Resultados}

No estado de Alagoas, conforme dados da Secretaria Nacional de Vigilância em Saúde, $\quad 73 \%$ das residências é abastecida por Fornecimento coletivo de água por meio de sistema de abastecimento de água (SAA); a parcela restante emprega soluções alternativas de abastecimento: solução alternativa coletiva (SAC) e solução alternativa individual (SAI) [Figura 1].

Figura 1 A. Cobertura de Abastecimento de Água por Forma De Abastecimento

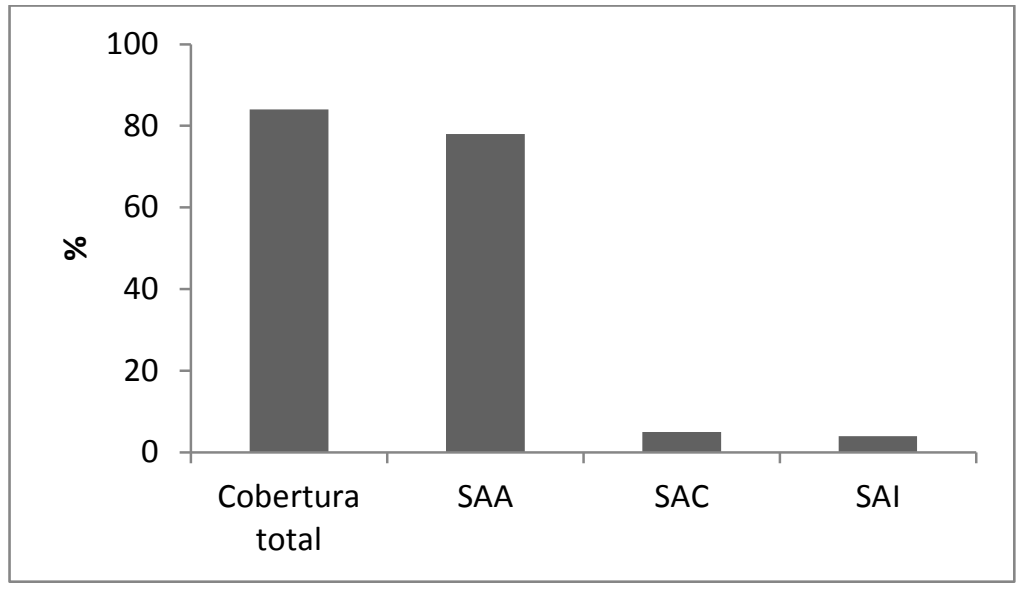

Fonte: SNVS ${ }^{(7)}$.

Figura 1B. Cobertura de Abastecimento de Água por Forma De Abastecimento

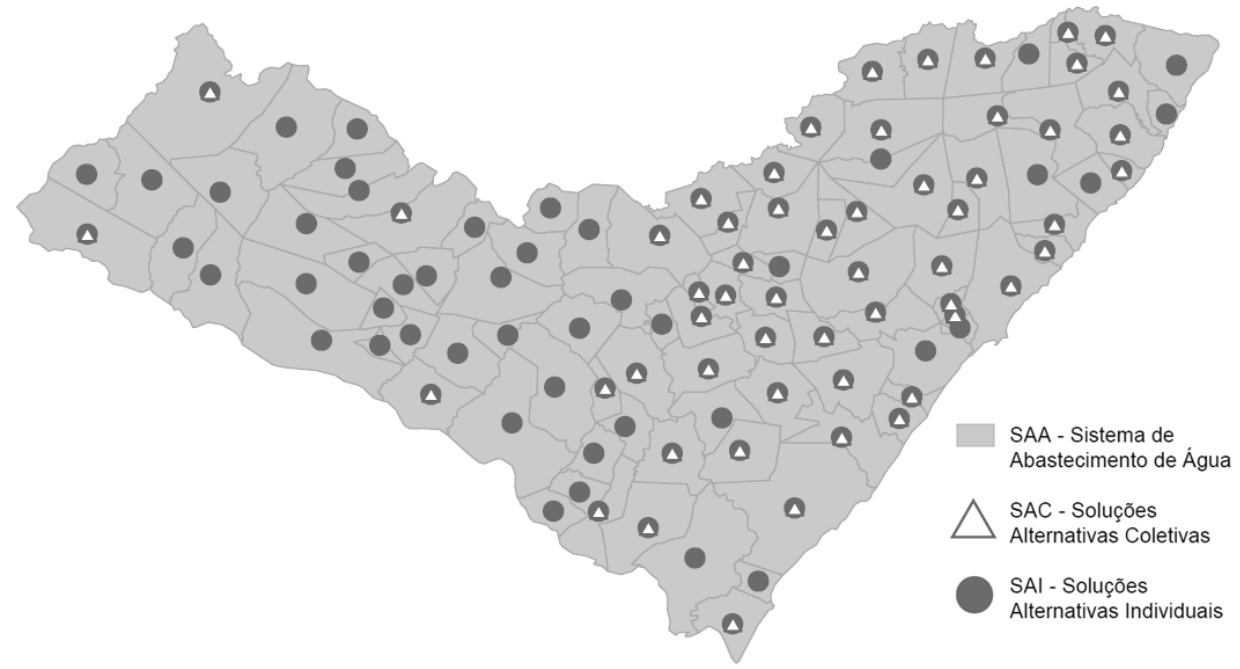

Fonte: $\operatorname{SNVS}^{(7)}$. 
Apesar da distribuição de água em Alagoas ser em sua maioria realizada por SAA isso não significa que essa população recebe água tratada. Conforme comparação do percentual da população abastecida por SAA [Figura $2^{\mathrm{a}}$ ]. o estado de Alagoas apresenta um quantitativo de população abastecida por SAA sem tratamento superior ao percentual da região Nordeste e três vezes maior que o cenário nacional.

\section{Figura 2A. Comparação do Percentual da População Abastecida por SAA Sem Tratamento e Taxas de Incidência de Hepatite A}

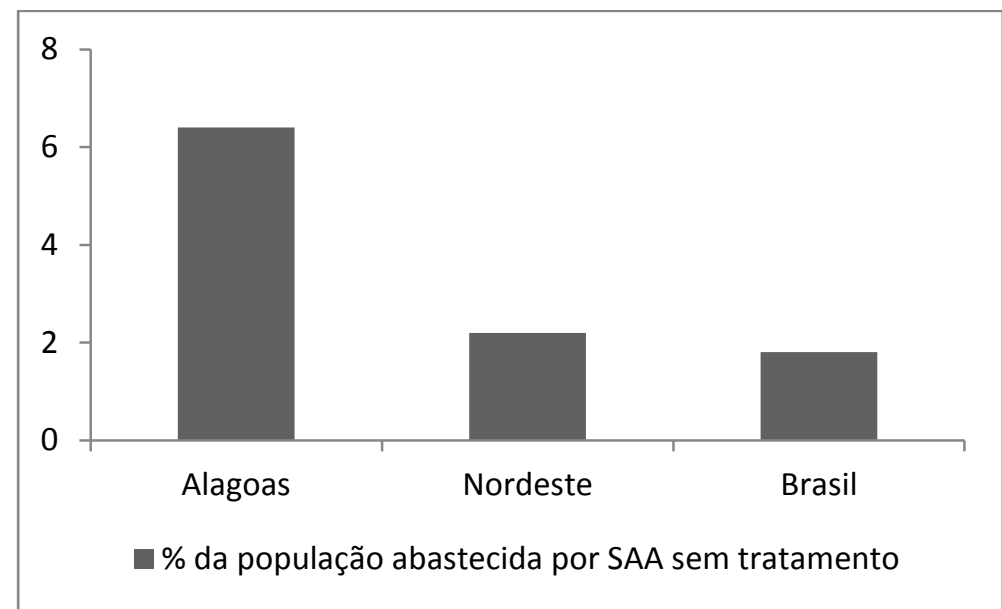

Fonte: $\mathrm{SNVS}^{(7)}$.

A Hepatite A é citada pela literatura especializada como uma das principais doenças de veiculação hídrica ${ }^{(10)}$. As taxas de incidência desta doença em
Alagoas foram superiores aos mesmos valores para a Região Nordeste e aqueles registrados para o país [Figura 2B].

Figura 2B. Comparação do Percentual da População Abastecida por SAA Sem Tratamento e Taxas de Incidência de Hepatite A

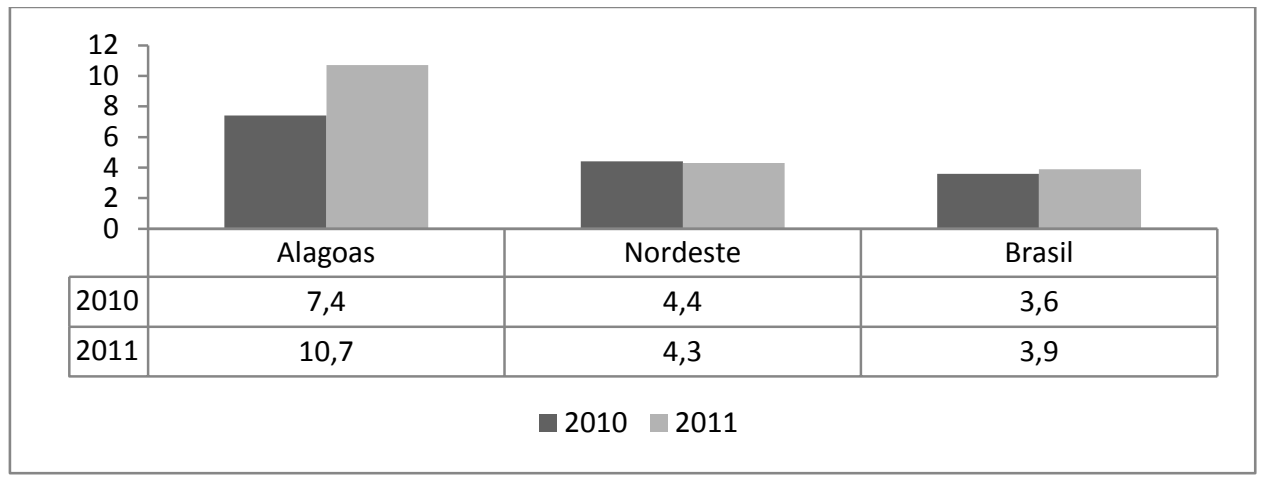

Fonte: $\mathrm{SNVS}^{(7)}$. 
Para o mesmo período, o IBGE básico no estado [Figura 3].

verificou baixa cobertura de saneamento

Figura 3. Percentual de Domicílios com Rede Geral de Abastecimento de Água e Esgotamento Sanitário

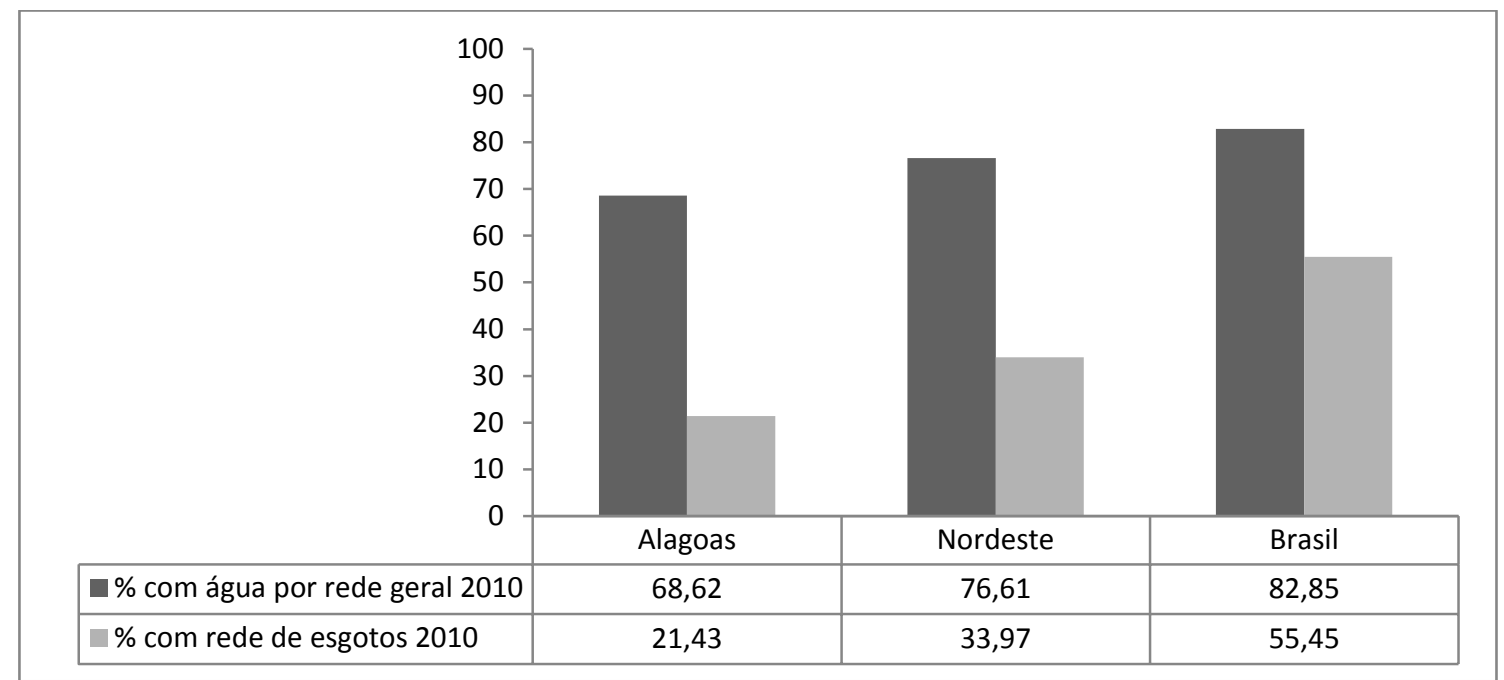

Fonte: $\mathrm{IBGE}^{(5)}$.

A Figura 4 apresenta a por SAA com água sem tratamento e distribuição da população abastecida que foi acometida por Hepatite A no território alagoano.

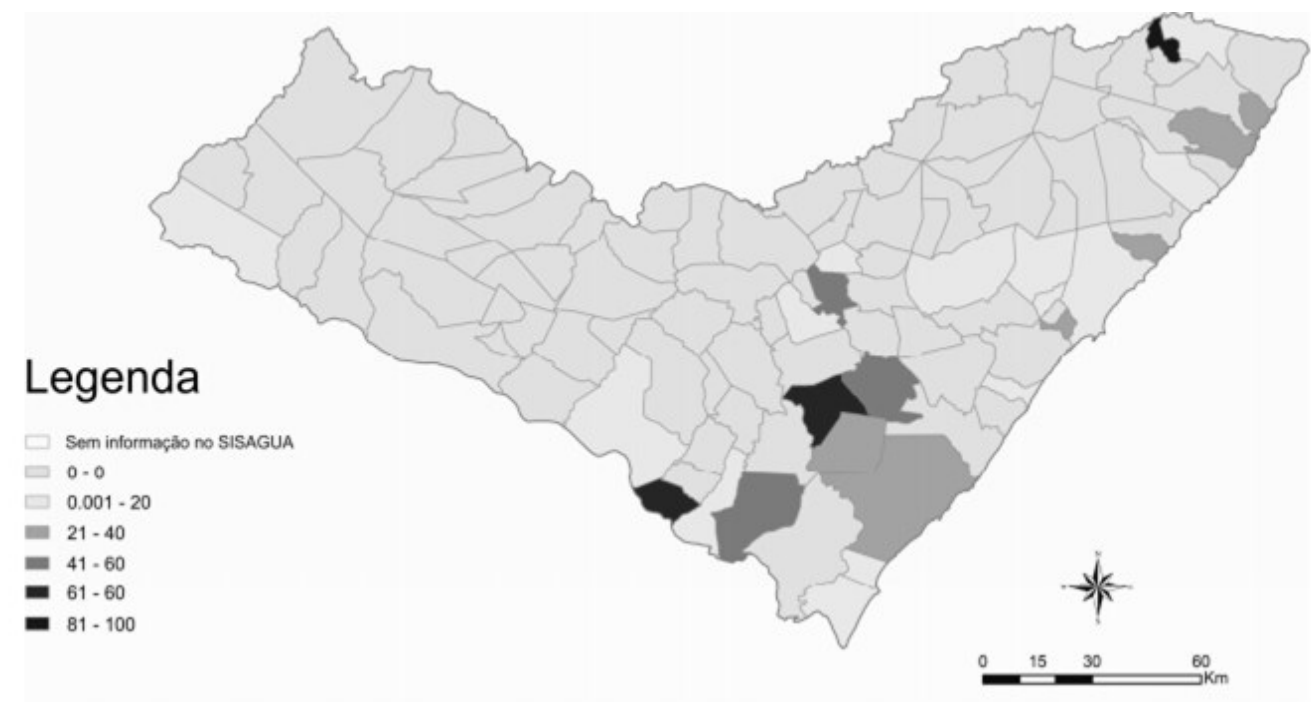

Fonte: $\operatorname{SNVS}^{(7)}$.

As Tabelas 1A e 1B apresentam dados relativos à epidemiologia da
Hepatite A. O ano de 2011, objeto de estudo dos dados deste trabalho, foi 
aquele que apresentou o maior número de casos confirmados da doença. O estado de Alagoas no período de 2010-
2013 apresentou média de taxa de incidência por Hepatite A que superou em quase $50 \%$ a média brasileira.

Tabela 1 A. Casos Confirmados de Hepatite A por Região de Saúde

\begin{tabular}{|c|c|c|c|c|}
\hline & 2010 & 2011 & 2012 & 2013 \\
\hline $1^{\mathrm{a}}$ Região de Saúde & 121 & 188 & 95 & 82 \\
\hline $2^{\mathrm{a}}$ Região de Saúde & 2 & 8 & 1 & 5 \\
\hline $3^{\text {a }}$ Região de Saúde & 8 & 29 & 17 & 5 \\
\hline $4^{\mathrm{a}}$ Região de Saúde & 14 & 5 & 4 & 2 \\
\hline $5^{\mathrm{a}}$ Região de Saúde & 25 & 24 & 2 & 4 \\
\hline $6^{\mathrm{a}}$ Região de Saúde & 2 & 17 & 0 & 1 \\
\hline $7^{\mathrm{a}}$ Região de Saúde & 21 & 20 & 8 & 50 \\
\hline $8^{\mathrm{a}}$ Região de Saúde & 8 & 7 & 0 & 7 \\
\hline 9ª Região de Saúde & 11 & 18 & 17 & 15 \\
\hline $10^{a}$ Região de Saúde & 18 & 3 & 1 & 39 \\
\hline TOTAL & 230 & 319 & 145 & 210 \\
\hline
\end{tabular}

Fonte: Datasus ${ }^{(8)}$.

Tabela 1 B. Taxa de Incidência de Hepatite A (por 100.000 habitantes) por Ano de Notificação

\begin{tabular}{c|ccccc}
\hline & 2010 & 2011 & 2012 & 2013 & MÉDIA \\
\hline Alagoas & 7,4 & 10,7 & 4,7 & 6,1 & 7.2 \\
Nordeste & 4,4 & 4,3 & 3,2 & 4,1 & 4.0 \\
Brasil & 3,6 & 3,9 & 3,4 & 3,1 & 3.5 \\
\hline
\end{tabular}

Fonte: Datasus ${ }^{(8)}$.

Dentre as análises de controle de qualidade estabelecidas pelo Ministério da Saúde para monitoramento da água para consumo humano, destacam-se determinação da quantidade de Cloro Residual Livre (CRL), Coliformes Totais (CLT) e Turbidez (TBD), definidas a seguir. A Diretriz Nacional de Amostragem da Vigilância da Qualidade da Água estabelece que um percentual de $45 \%$ de amostras obrigatórias para as análises de CRL, CLT e TBD ${ }^{(11)}$. Para os 2010 e 2011 as amostras realizadas pelo setor de vigilância em saúde de Alagoas estão abaixo dos preconizado pela legislação [Figura 5].

Do quantitativo de análises realizadas, verifica-se que o percentual de amostras em conformidade com a Portaria MS 2914/2011 para o SAA é elevado $^{(12)}$. 
Figura 5. Percentual de Amostras Realizadas pela Vigilância, Conforme Estabelecido Pela Diretriz Nacional

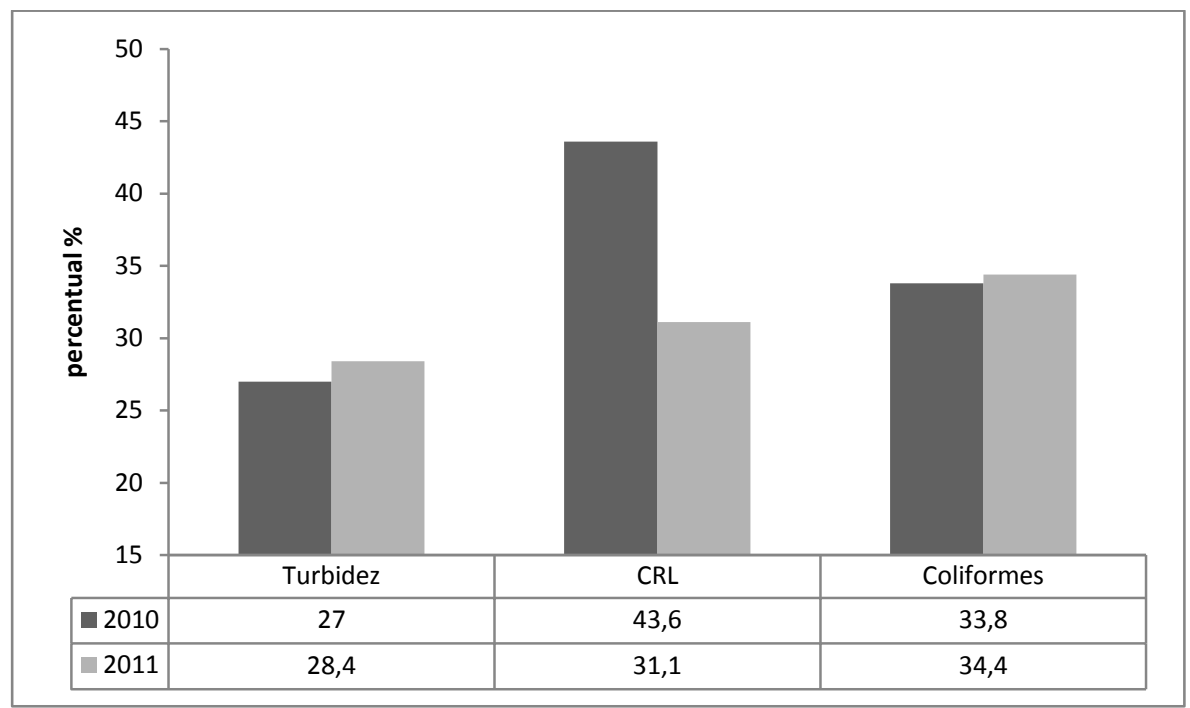

Fonte: $\mathrm{SNVS}^{(7)}$.

Para as soluções alternativas apenas o parâmetro TBD atingiu índices satisfatórios.
Quanto aos parâmetros CRL e CLT, ambos estão bem abaixo do ideal [Tabela 2].

Tabela 2. Levantamento (\%) de Amostras Realizadas por Tipo de Abastecimento em Conformidade com o Padrão de Potabilidade

\begin{tabular}{cccc}
\hline \multirow{2}{*}{ Parâmetros } & \multicolumn{2}{c}{ \% Amostras em Conformidade com o padrão de potabilidade } \\
& SAA & SAC & SAI \\
\hline TBD & 76,59 & 86,41 & 85,1 \\
CRL & 84,56 & 20,55 & 62,41 \\
CLT & 71,38 & 29,31 & 20,7 \\
\hline
\end{tabular}

Fonte: SVS/SISAGUA ${ }^{(8)}$.

O estado de Alagoas não realizou o monitoramento de cianobactérias, nem a pesquisa de agrotóxicos no ano em estudo. A legislação, por sua vez, estabelece que os prestadores de serviço devem realizar análises mensais quando a densidade de cianobactérias no manancial de abastecimento de água for $\leq 10.000$ cél $/ \mathrm{mL}$, e semanais, quando a densidade de cianobactérias for $\leq 10.000$ cél $/ \mathrm{mL}^{(12)}$. O monitoramento de agrotóxicos é obrigatório no país para toda água a ser empregada no consume humano. 


\section{Discussão}

O objetivo principal para o estabelecimento de requisitos no que diz respeito ao monitoramento e verificação da qualidade da água fornecida à população é a proteção à saúde pública e a prevenção de doenças cuja incidência estão relacionadas ao tratamento inadequado deste recurso $^{(3,11,13)}$.

Os critérios adotados para assegurar essa qualidade têm por finalidade fornecer uma base para o incremento de estratégias de saúde pública que, se propriamente implementadas, garantirão a segurança do fornecimento desse recurso de forma segura para o consumo, não apresentando riscos à saúde da população $^{(13)}$.

A implementação de métodos visando o monitoramento da qualidade da água abastecida pelo poder público e pelas companhias de abastecimento é uma orientação fornecida pela Organização Mundial da Saúde - OMS, para todas as nações ${ }^{(3)}$.

No Brasil, ações de avaliação da qualidade da água para consumo humano são desenvolvidas pelo Ministério da Saúde e das Secretarias Municipais e Estaduais, através do Sistema Único de Saúde - SUS ${ }^{(11)}$.
Essas ações se justificam diante dos resultados apontados pelos índices de doenças e mortes que estão afetando a sociedade, ocasionadas por enfermidades de veiculação hídrica, a exemplo das doenças diarreicas, caracterizadas pela ingestão de água contaminada por meio de microorganismos.

Em Alagoas este monitoramento é estabelecido pela Portaria $n^{\circ}$ 2.914/2011, do Ministério da Saúde, que regula os procedimentos de controle e vigilância da qualidade da água para consumo humano e seu padrão de potabilidade no âmbito nacional ${ }^{(12)}$.

A proteção de contaminações externas ao processo de distribuição de água é a mais eficaz estratégia de garantia da saúde dos indivíduos. Muitos autores defendem que essa é a melhor forma de assegurar um recurso adequado para consumo humano e comercial $^{(14)}$. Falhas na proteção e no tratamento efetivo expõem a comunidade a riscos de doenças intestinais e a outras doenças infecciosas $^{(15)}$.

Os riscos à saúde relacionados com a água podem ser classificados em dois grupos, são eles: 1) riscos biológicos, relacionados ao consumo de água contaminada por vírus, bactérias e vermes em geral e que são responsáveis 
por casos de enterites, diarreias, Hepatite A, amebíase, entre outras; 2) relacionados à contaminação por poluentes, substâncias química diversas, resíduos de esgotos e sujidades em geral. Isso se torna uma preocupação, pois sabemos que além dos microrganismos provenientes da natureza, existem, também, fatores relacionados ao uso e descartes de substâncias pela população ${ }^{(15)}$.
A Diretriz Nacional de Vigilância da Qualidade da Água para Consumo Humano estabelece um conjunto de requisitos de análises que têm como finalidade detectar possíveis inconformidades na água a ser consumida pela população, as principais análises preconizadas, suas características e relevância $^{(11)}$, descritas na Tabela 3.

\section{Quadro 1. Análises de Qualidade Definidos na Diretriz Nacional de Amostragem da Vigilância da Qualidade da Água}

\begin{tabular}{|c|l|}
\hline Análise & \multicolumn{1}{c|}{ Descrição } \\
\hline \multirow{3}{*}{$\begin{array}{c}\text { Cloro Residual Livre } \\
\text { (CRL) }\end{array}$} & $\begin{array}{l}\text { O cloro é um produto químico utilizado na desinfecção da água com o fim de } \\
\text { eliminar agentes patogênicos causadores de doenças, tais como bactérias, vírus e } \\
\text { protozoários e são os causadores de doenças como cólera, febre tifoide, disenteria e } \\
\text { Hepatite B. Sua medida serve para controlar a dosagem que está sendo aplicada e } \\
\text { também para acompanhar sua evolução durante o tratamento. A Portaria no } \\
2.914 / 2011 \text { determina a obrigatoriedade de se manter, no mínimo, 0,2 mg/L de } \\
\text { cloro residual livre ou 2 mg/L de cloro residual combinado em toda a extensão do } \\
\text { sistema de distribuição. }\end{array}$ \\
\hline \multirow{5}{*}{$\begin{array}{c}\text { Coliformes Totais } \\
\text { (CLT) }\end{array}$} & $\begin{array}{l}\text { Pelo estudo da sua concentração na água pode-se estabelecer um parâmetro } \\
\text { indicador da existência de possíveis microrganismos patogênicos, que são } \\
\text { responsáveis pela transmissão de diversas doenças. Os coliformes totais são } \\
\text { bactérias do grupo coliforme, bacilos gram-negativos, aeróbios ou anaeróbios } \\
\text { facultativos, não formadores de esporos, oxidase-negativos, capazes de desenvolver } \\
\text { a 35,0 } \pm 0,50 C \text { em 24-48 horas. Pertence a esse grupo bactérias do dos gêneros } \\
\text { Escherichia, Citrobacter, Klebsiella e Enterobacter. }\end{array}$ \\
\hline $\begin{array}{c}\text { Turbidez } \\
\text { dissolvidos no fluído e cuja presença altera as suas propriedades ópticas. Um } \\
\text { aumento na turvação da água é geralmente ocasionado pelo crescimento de } \\
\text { bactérias e leveduras em suspensão, razão pela qual este parâmetro é um excelente } \\
\text { indicador sanitário da presença destes microrganismos na água. }\end{array}$ \\
\hline
\end{tabular}

Fonte: Adaptado de FUNASA ${ }^{(3)}$.

Em Alagoas, o controle de qualidade de água para ao consumo humano, desde os sistemas produtores aos sistemas de distribuição, é realizado por empresas locais responsáveis pelo saneamento, e em alguns casos pela prefeitura do município, sendo monitorado pela Secretaria Estadual de Saúde (SESAU). 
No estado, o abastecimento de água à população ocorre de três formas distintas: fornecimento coletivo de água através de sistema de abastecimento de água (SAA); abastecimento coletivo de água através de solução alternativa coletiva (SAC); abastecimento individual através de solução alternativa individual (SAI).

27\% dos usuários em Alagoas são atendidos por SAC e/ou SAI sendo a maioria absoluta dos usuários atendida por SAA $(73 \%)$.

A Portaria MS no 2.914/2011, em seus artigos $3^{\circ}$ e $4^{\circ}$, determina que toda água destinada ao consumo humano, distribuída coletivamente através de SAA ou SAC, deve ser objeto de controle e vigilância ${ }^{(12)}$. No âmbito local, contudo, registrou-se um percentual de $6,4 \%$ da população abastecida por SAA sem tratamento, e no último Censo do Instituto Brasileiro de Geografia e Estatística (IBGE) foi verificado que existe baixa cobertura de saneamento básico em Alagoas [Figura $3]^{(5)}$.

Assim, apesar de não ter sido identificada relação direta entre as taxas de morbidade por Hepatite A na população apresentada e as condições de abastecimento das regionais de saúde do estado, a literatura especializada aponta que a Hepatite A tem maior prevalência em áreas com más condições sanitárias e higiênicas e em localidades onde prevalecem os sistemas de abastecimento de água sem tratamento $^{(10,17)}$.

No entanto, é preciso verificar os demais fatores que levam ao aumento dessas doenças nas regiões que possuem precárias infraestruturas urbanas e sanitárias, como o descarte de resíduos pela população e a coleta de lixo nos bairros, que podem não são as principais causas, porém contribuem para a contaminação da água e aumento das doenças ${ }^{(10,17)}$.

Outro provável fator que pode ter contribuído significativamente para as elevadas taxas de morbidade pela doença pode ser o próprio percentual de domicílios abastecidos por SAA sem tratamento.

\section{Conclusão}

Diante desses resultados, fica evidente a necessidade de fortalecimento das ações do setor de saúde e dos responsáveis pelos sistemas de fornecimento no sentido de implantar as diretrizes em vigor e aperfeiçoar o monitoramento dos parâmetros de controle, a fim de tornar o recurso fornecido à população da região nordeste mais seguro e com qualidade 
suficiente para prevenção de agravos diversos de veiculação hídrica.

Os dados aqui expostos reforçam também a importância de novos estudos sobre a temática, tendo em vista possíveis cenários de mudanças em ações de controle e monitoramento e a necessidade de se conhecer em profundidade, atualizar e ratificar as informações aqui presentes, além de suscitar a necessidade de estratégias de promoção de saúde e controle adequado dos parâmetros de potabilidade por meio de uma da articulação entre os núcleos de saúde dos governos e dos setores que fazem parte dos sistemas públicos e privados de distribuição de água, além de demais órgãos interessados nesse monitoramento.

\section{Referências}

1. Daniel MHB, Cabral AR. A Vigilância da qualidade da água para consumo humano (VIGIAGUA) e os objetivos do desenvolvimento do milênio (ODM). Cadernos Saúde Coletiva 2011; 19(4):487-92.

\section{Bergamasco}

AMDD.

Contaminantes químicos em águas destinadas ao consumo humano no Brasil. Cadernos Saúde Coletiva 2011;19(4):479-86.

3. World Health Organization. Guidelines for drinking-water quality. 4. ed. Geneva: OMS; 2011.
4. Frazão P, Peres MA, Cury JA. Qualidade da água para consumo humano e concentração de fluoreto. Rev. Saúde Publica. 2011;45(5):96473.

5. Instituto Brasileiro de Geografia e Estatística (BR). Banco de dados do Censo 2010. Brasília: IBGE; 2010.

6. Companhia de Abastecimento e Saneamento do Estado de Alagoas. Sistemas Coletivos. [internet]. Disponível em: http://www.casal.a 1.gov.br/sistema-coletivo.

7. Ministério da Saúde (BR). Secretaria Nacional de Vigilância em Saúde. Relatório de avaliação da Vigilância da Qualidade da Água no Estado de Alagoas (Ano base 2011). Brasília: Ministério da Saúde; 2012.

8. Ministério da Saúde (BR). DATASUS. Hepatites Virais 20102013. Brasília: Ministério da Saúde; 2017.

9. Ministério da Saúde (BR). Sistema de Informação de Vigilância da Qualidade da Água para consumo humano. Brasília: Ministério da Saúde; 2017.

10. Ministério da Saúde (BR). Secretaria de Vigilância em Saúde. Guia de Vigilância em Saúde. 2. ed. Brasília: Ministério da Saúde; 2017.

11. Ministério da Saúde (BR). Diretriz Nacional do Plano de Amostragem da Vigilância da Qualidade da Água para Consumo Humano. Brasília: Ministério da Saúde; 2016. 
12. Ministério da Saúde (BR). Portaria 2.914, de 12 de dezembro de 2011. Dispõe sobre os procedimentos de controle e de vigilância da qualidade da água para consumo humano e seu padrão de potabilidade. Brasília: Ministério da Saúde; 2012.

13. Ministério da Saúde (BR). Manual de vigilância e controle da qualidade da água para consumo humano. Brasília: Ministério da Saúde; 2006.

14. Queiroz ACL, Cardoso LSM, Silva SCF. Programa Nacional de Vigilância em Saúde Ambiental Relacionada à Qualidade da Água para Consumo Humano (VIGIAGUA): lacunas entre a formulação do programa e sua implantação na instância municipal. Saúde Soc. 2012; 21(2):465-78.

15. Neves DP. Parasitologia Humana. 13. ed. São Paulo: Atheneu ; 2016.

16. Fundação Nacional de Saúde (BR). Manual de análise de água. 4 ed. Brasília: Ministério da Saúde; 2013.

17. Fonseca, JCF. Histórico das hepatites virais. Rev da Sociedade Brasileira de Medicina Tropical. 2010; 43(3):322-30. 